\title{
DYNAMIC SEMI-SUPERVISED FUZZY CLUSTERING FOR DENTAL X-RAY IMAGE SEGMENTATION: AN ANALYSIS ON THE ADDITIONAL FUNCTION
}

\author{
TRAN MANH TUAN ${ }^{1}$, LE HOANG SON ${ }^{2}$, LE BA DUNG ${ }^{3}$ \\ ${ }^{1}$ School of Information and Communication Technology, \\ Thai Nguyen University; \\ tmtuan@ictu.edu.vn \\ ${ }^{2}$ VNU University of Science, Vietnam National University; \\ sonlh@vnu.edu.vn \\ ${ }^{3}$ Institute of Information Technology, VAST; \\ lbdung@ioit.ac.vn
}

\begin{abstract}
Dental X-ray image segmentation is a necessary and important process in medical diagnosis, which assists clinicians to make decisions about possible dental diseases of a patient from a dental X-ray image. It is a multi-objective optimization problem which involves basic components of fuzzy clustering, spatial structures of a dental image, and additional information of experts expressed through a pre-defined membership matrix. In our previous work, the authors presented a semi-supervised fuzzy clustering algorithm using interactive fuzzy satisficing named as SSFC-FS for this problem. An important issue of SSFC-FS is that the pre-defined membership matrix is a fixed function in the sense that it uses the same structure and parameters for all dental images. This is a shortcoming of SSFC-FS since each image has its own structure and morphology so that it needs different membership matrices. In this paper, the authors propose another new dynamic semi-supervised fuzzy clustering called SSFC-FSAI that extends SSFC-FS by employing a collection of pre-defined membership matrices for dental images. A procedure to choose a suitable pre-defined membership matrix for a given dental X-ray image is proposed and attached to SSFC-FSAI. Experimental results on a real dataset of 56 dental X-ray images from Hanoi University of Medical in 2014 - 2015 show that SSFC-FSAI has better accuracy than SSFC-FS and the relevant algorithms.
\end{abstract}

Keywords. Additional function, dynamic semi-supervised fuzzy clustering, dental X-ray image segmentation, fuzzy C-Means.

\section{INTRODUCTION}

One of the most increasing concerns in medical science especially medical informatics in recent years is the medical diagnosis by computerized methods, which assist clinicians to make decisions about possible diseases of a patient from his syndromes or X-ray images. In dentistry, this matter is 
much more important since dentists often use their own experiences combined with information from medical devices such as dental X-ray images to judge the patient's diseases and therapies. This makes meaning for inexperienced clinicians who are able to advance their professional by learning from real cases. It is possible that successful cases are stored in the system as clinical knowledge which is later re-used and learnt by other clinicians in next cases. Such the diagnosis system would be an efficient computerized tool that supports clinicians' professional effectively.

The dental X-ray image segmentation is a necessary and important process in medical diagnosis. The aim of segmentation is to create several distinct groups in a dental X-ray image whereas pixels in a group have more similarity than those in other groups. The dental image can be classified by various areas namely background and dental structures or by disease and non-disease parts. Those areas are then compared with standard disease patterns by a fast search method to identify whether or not the dental image contains any disease. Therefore, the accuracy of segmentation is quite important to successful decisions of diseases for a patient. This problem has been studied extensively in [1-15] showing that the typical and popular methods are the Otsu thresholding method [11], Fuzzy CMeans (FCM) clustering [1], and Semi-Supervised Entropy regularized Fuzzy Clustering (eSFCM) algorithm [14]. Nevertheless, those methods faced the problems of threshold value determination, determining common boundaries of clusters, and lacking of spatial structures of an X-ray image.

The dental X-ray image segmentation can be formulated by a multi-objective optimization problem which involves basic components of fuzzy clustering, spatial structures of a dental image, and additional information of experts expressed through a pre-defined membership matrix. From this point of view, our previous work in [16] presented a semi-supervised fuzzy clustering algorithm using interactive fuzzy satisficing named as SSFC-FS for this problem. SSFC-FS solves each single optimization problem by Lagrange method and then constructs an initial global solution from the single ones. The initial solution is improved in each iteration step until the stopping condition holds. SSFC-FS has better accuracy than Otsu, FCM and eSFCM as experimentally validated on a real dataset of 56 dental X-ray images from Hanoi University of Medical in the period $2014-2015$.

A drawback of SSFC-FS is that the pre-defined membership matrix is a fixed function in the sense that it uses the same structure and parameters for all dental images. This is a shortcoming of SSFC-FS since each image has its own structure and morphology so that it needs different membership matrices. Thus in this paper, a new dynamic semi-supervised fuzzy clustering algorithm called SSFC-FSAI is proposed to handle the problem of SCFC-FS regarding the additional function in term of pre-defined membership matrix. Specifically, the authors define a collection of pre-defined membership matrices for dental images, and then propose a procedure to choose a suitable membership matrix for a given image. Experimental results on a real dataset of 56 dental X-ray images from Hanoi University of Medical will be done to validate the accuracy of SSFC-FSAI in comparison with SSFC-FS and other relevant algorithms.

This paper is organized as following: the background results [16] are presented in Section 2. These include modeling of the dental X-ray image segmentation problem in form of semi-supervised fuzzy clustering and details of the SSFC-FS method. The ideas and details of the new method SSFC-FSAI are stated in Section 3. Section 4 gives the implemented evaluation of SSFC-FSAI. Lastly, Section 5 gives conclusions and delineates some continuing works in the future. 


\section{BACKGROUND}

The dental X-ray image segmentation problem in form of semi-supervised fuzzy clustering is shown as follows.

$$
\begin{aligned}
J= & \sum_{k=1}^{N} \sum_{j=1}^{C} u_{k j}^{2}\left\|X_{k}-V_{j}\right\|^{2}+\sum_{k=1}^{N} \sum_{j=1}^{C} u_{k j}^{m} R_{k j}^{2} \\
& +\sum_{k=1}^{N} \sum_{j=1}^{C} u_{k j}^{m} \frac{1}{l} \sum_{i=1}^{l} w_{k i}+\sum_{k=1}^{N} \sum_{j=1}^{C}\left|u_{k j}-\bar{u}_{k j}\right|^{m}\left\|X_{k}-V_{j}\right\| \rightarrow \min \\
& \sum_{j=1}^{C} u_{k j}=1 ; \quad u_{k j} \in[0,1] ; \forall k=\overline{1, N},
\end{aligned}
$$

where the pre-defined membership matrix $\bar{u}_{k j}$ satisfies the conditions:

$$
\sum_{j=1}^{C} \bar{u}_{k j} \leq 1 ; \quad u_{k j} \in[0,1] ; \quad \forall k=\overline{1, N}
$$

In those formulae, meanings of the parameters are:

- $m$ is the fuzzier $(m>0)$;

- $C$ is the number of clusters;

- $N$ is the number of data point;

- $r$ is the dimension of data;

- $u_{k j} \in[0,1]$ is the membership degree of $\mathrm{k}^{\text {th }}$ data point to the $j^{\text {th }}$ cluster, $j=1, \ldots, C$, $k=1, \ldots, N$

- $X_{k} \in R^{r}$ is the $\mathrm{k}^{\text {th }}$ data element of data set $X=\left\{X_{1}, X_{2}, \ldots, X_{N}\right\}$;

- $V_{j}$ is center of $j^{\text {th }}$ cluster, $j=1, \ldots, C$;

- $l$ : the number of features;

- $w_{k i}$ : weight of $\mathrm{i}^{\text {th }}$ feature in $k^{\text {th }}$ data point, $k=1, \ldots, N, i=1, \ldots, l$;

- $R_{k j}$ : spatial distance function between $X_{k}$ and $V_{j}, k=1, \ldots, N, j=1, \ldots, C$.

In [15], the authors use the function below for $\bar{u}_{k j}$.

$$
\bar{u}_{k j}=\left\{\begin{array}{lll}
\alpha u_{1}, & \text { when } & u_{1} \geq u_{2} \\
\alpha u_{2}, & \text { when } & u_{1}<u_{2}
\end{array} \quad, \alpha \in[0,1], j=\overline{1, C}, k=\overline{1, N}\right.
$$

Where $u_{1}$ is defined from the final membership matrix of FCM and $u_{2}$ is defined from spatial features of the dental image. 


$$
u_{2}=\frac{\sum_{i=1}^{l} w_{i}}{\max \left\{\sum_{i=1}^{l} w_{i}\right\}} .
$$

It is possible to separate the objective function (1) into three independent functions and use Interactive Fuzzy Satisficing [17] method to find out the global solution. This was presented in the SSFC-FS algorithm which is described as follows.

$J_{1}$ is the standard objective function of Fuzzy C-Means (FCM) clustering method. It is formulated by equation (6) in order to minimize the distances between clusters' center and data points. $J_{2}$ represents the spatial information of dental image. $J_{3}$ defines the additional information of semisupervised fuzzy clustering methods.

Initialization: Solve the following subproblems by Lagrange method:

- Problem 1: $\min \left\{J_{1}(u), u \in R^{C \times N}\right.$ satisfies $\left.(2)\right\}$.

Denote $d_{k j}=\left\|X_{k}-V_{j}\right\|^{2}, k=1, \ldots, N ; j=1, \ldots, C$. The objective function $J_{1}$ is:

$$
J_{1}=\sum_{k=1}^{N} \sum_{j=1}^{C} u_{k j}^{m} d_{k j} .
$$

- Problem 2: $\min \left\{J_{2}(u), u \in R^{C \times N}\right.$ satisfies (2) $\}$.

Let $\alpha_{k j}=R_{k j}^{2}+\frac{1}{l} \sum_{i=1}^{l} w_{k i}, k=1, \ldots, N ; j=1, \ldots, C$, we have:

$$
J_{2}=\sum_{k=1}^{N} \sum_{j=1}^{C} u_{k j}^{m} \alpha_{k j} .
$$

- Problem 3: $\min \left\{J_{3}(u), u \in R^{C \times N}\right.$ satisfies (2) $\}$ with the objective function $J_{3}$ being written as,

$$
J_{3}=\sum_{k=1}^{N} \sum_{j=1}^{C}\left|u_{k j}-\bar{u}_{k j}\right|^{m} d_{k j} .
$$

\begin{tabular}{|c|c|c|c|}
\hline $\begin{array}{ll} & \text { Objective functions } \\
\text { Solutions } & \\
\end{array}$ & $J_{1}$ & $J_{2}$ & $J_{3}$ \\
\hline$u_{j k}^{1}$ & $z_{11}$ & $z_{12}$ & $z_{13}$ \\
\hline$u_{j k}^{2}$ & $z_{21}$ & $z_{22}$ & $z_{23}$ \\
\hline$u_{j k}^{3}$ & $z_{31}$ & $z_{32}$ & $z_{33}$ \\
\hline
\end{tabular}

Assuming that optimal solutions of the sub-problems are $u_{k j}^{1}, u_{k j}^{2}, u_{k j}^{3}$. Set up a pay-off table 1 :

Table 1: Pay-off table

Denote that:

$$
\begin{aligned}
& \underline{z}_{i}=\min \left\{z_{t i}, t=1,2,3\right\}, \quad \bar{z}_{i}=\max \left\{z_{t i}, t=1,2,3\right\}, \quad i=1,2,3, \\
& S_{p}=\left\{u^{1}, u^{2}, u^{3}\right\}, r=1, a_{i}^{(r)}=\underline{z}_{i} .
\end{aligned}
$$




\section{Iterative steps:}

Step 1: Fuzzy satisficing functions for each of subproblems are defined by,

$$
\mu_{i}\left(J_{i}\right)=\frac{J_{i}-\underline{z}_{i}}{\bar{z}_{i}-\underline{z}_{i}}, i=1,2,3
$$

Based on these functions, there is the combination satisficing function:

$$
\begin{array}{r}
Y=b_{1} \mu_{1}\left(J_{1}\right)+b_{2} \mu_{2}\left(J_{2}\right)+b_{3} \mu_{3}\left(J_{3}\right) \rightarrow \min \\
b_{1}+b_{2}+b_{3}=1 \quad 0 \leq b_{1}, b_{2}, b_{3} \leq 1
\end{array}
$$

Then the optimal problem is solved with the objective function as in (12) and the constraints including original constraints (2) and some more constraints below.

$$
J_{i}(x) \geq a_{i}^{(r)}, i=1,2,3 \cdots
$$

The objective function of this problem can be rewritten as,

$$
Y=\frac{b_{1}}{\bar{z}_{1}-\underline{z}_{1}} J_{1}+\frac{b_{2}}{\bar{z}_{2}-\underline{z}_{2}} J_{2}+\frac{b_{3}}{\bar{z}_{3}-\underline{z}_{3}} J_{3}-\left(\frac{b_{1} \underline{z}_{1}}{\bar{z}_{1}-\underline{z}_{1}}+\frac{b_{2} \underline{z}_{2}}{\bar{z}_{2}-\underline{z}_{2}}+\frac{b_{3} \underline{z}_{3}}{\bar{z}_{3}-\underline{z}_{3}}\right)
$$

Taking derivative of (15) yields

$$
\frac{\partial Y}{\partial u_{j k}}=\frac{b_{1}}{\bar{z}_{1}-\underline{z}_{1}} \frac{\partial J_{1}}{\partial u_{j k}}+\frac{b_{2}}{\bar{z}_{2}-\underline{z}_{2}} \frac{\partial J_{2}}{\partial u_{j k}}+\frac{b_{3}}{\bar{z}_{3}-\underline{z}_{3}} \frac{\partial J_{3}}{\partial u_{j k}}+\eta_{k}, j=\overline{1, C}, k=\overline{1, N} .
$$

For each of sets $\left(b_{1}, b_{2}, b_{3}\right)$ satisfying (13), it results in an optimal solution $u^{(r)}=\left(u_{k j}^{(r)}\right)_{C \times N}$. Step 2:

- If $\mu_{\min }=\min \left\{\mu_{i}\left(J_{i}\right), i=1, \ldots, 3\right\}>\theta$, with $\theta$ is an optional threshold then $u^{(r)}$ is not acceptable. Otherwise, if $u^{(r)} \notin S_{p}$ then $u^{(r)}$ is put on $S_{p}$.

- In the case of needing to expand $S_{p}$, set $r=r+1$ and check the conditions:

If $r>L_{1}$ or after $L_{2}$ consecutive iterations that $S_{p}$ is not expanded ( $L_{1}, L_{2}$ has optional values) then set $a_{i}^{(r)}=\underline{z}_{i}, i=1,,_{2}, 3$ and get a randomly index $h$ in $\{1,2,3\}$ to put $a_{h}^{(r)} \in\left[\underline{z}_{h}, \bar{z}_{h}\right)$. Then return to step1.

- In the case of not needing to expand $S_{p}$ then go to step 3.

Step 3: Rejecting dominant solutions from $S_{p}$. End of process. 
Lemma 1. With a given parameter set $\left(b_{1}, b_{2}, b_{3}\right)$, the solutions of (15) are:

$$
\begin{aligned}
u_{k j}^{(r)}= & \frac{\frac{b_{3}^{(r)}}{\bar{z}_{3}-\underline{z}_{3}} \times d_{k j} \times \bar{u}_{k j}-\frac{\eta_{k}^{(r)}}{2}}{\left(\frac{b_{1}^{(r)}}{\bar{z}_{1}-\underline{z}_{1}}+\frac{b_{3}^{(r)}}{\bar{z}_{3}-\underline{z}_{3}}\right) d_{k j}+\frac{b_{2}^{(r)}}{\bar{z}_{2}-\underline{z}_{2}} \times \alpha_{k j}}, k=\overline{1, N}, j=\overline{1, C} \\
\eta_{k}^{(r)}= & 2 \times \frac{\sum_{j=1}^{C} \frac{\frac{b_{3}^{(r)}}{\bar{z}_{3}-\underline{z}_{3}} \times d_{k j} \times \bar{u}_{k j}}{\left(\frac{b_{1}^{(r)}}{\bar{z}_{1}-\underline{z}_{1}}+\frac{b_{3}^{(r)}}{\bar{z}_{3}-\underline{z}_{3}}\right) d_{k j}+\frac{b_{2}^{(r)}}{\bar{z}_{2}-\underline{z}_{2}} \times \alpha_{k j}}-1}{\sum_{j=1}^{C} \frac{1}{\left(\frac{b_{1}^{(r)}}{\bar{z}_{1}-\underline{z}_{1}}+\frac{b_{3}^{(r)}}{\bar{z}_{3}-\underline{z}_{3}}\right) d_{k j}+\frac{b_{2}^{(r)}}{\bar{z}_{2}-\underline{z}_{2}} \times \alpha_{k j}}}, k=\overline{1, N} . \\
V_{j}^{(r)}= & \frac{\sum_{k=1}^{N}\left(\frac{b_{1}^{(r)}}{\bar{z}_{1}-\underline{z}_{1}} \times\left(u_{k j}^{(r)}\right)^{2}+\frac{b_{3}^{(r)}}{\bar{z}_{3}-\underline{z}_{3}}\left(u_{k j}^{(r)}-\bar{u}_{k j}\right)^{2}\right) X_{k}}{\sum_{k=1}^{N}\left(\frac{b_{1}^{(r)}}{\bar{z}_{1}-\underline{z}_{1}} \times\left(u_{k j}^{(r)}\right)^{2}+\frac{b_{3}^{(r)}}{\bar{z}_{3}-\underline{z}_{3}}\left(u_{k j}^{(r)}-\bar{u}_{k j}\right)^{2}\right)} .
\end{aligned}
$$

Base on the solutions, SSFC-FS determines segmented areas in a dental X-ray image where predefined membership matrix $\bar{u}_{k j}$ is fixed as in equations $(4,5)$.

\section{THE SSFC-FSAI METHOD}

This section presents the main parts of this study. The SSFC-FSAI method is demonstrated in Section 3.1. A collection of pre-defined membership matrices for dental images is proposed in Section 3.2. Section 3.3 shows a procedure to choose a suitable membership matrix for a given image. Lastly, some advantages of proposed method are given in Section 3.4.

\subsection{The SSFC-FSAI method}

Figure 1 illustrates the main mechanism of SSFC-FSAI. The input of this method is a dental X-ray image which is then segmented by FCM and extracted feature information. From the achieved results, a suitable pre-defined membership matrix and its parameters are automatically estimated for a given dental image. This function is then used to calculate the final outputs of the semi-supervised fuzzy clustering model as in Section 2. The segmented image is then evaluated using various criteria.

\subsection{Defining a collection of membership matrices}

In this section, some additional information functions are introduced as follows.

a) Gaussian function:

$$
G_{k j}=e^{-\frac{1}{2}\left(\frac{\left\|x_{k}-v_{j}\right\|}{\max _{i=1, N}\left\{\left\|x_{i}-v_{j}\right\|\right\}}\right)^{2}}, \quad j=\overline{1, C}, k=\overline{1, N}
$$

where $x_{j}$ : is the data of $j^{\text {th }}$ pixel, $v_{i}$ : is the center of $\mathrm{i}^{\text {th }}$ cluster (defined from FCM)

$$
\bar{u}_{k j}=\alpha \frac{G_{k j}}{\sum_{i=1}^{C} G_{k i}+\min _{i=1, C}\left\{G_{k i}\right\}}, \alpha \in[0,1], j=\overline{1, C}, k=\overline{1, N}
$$




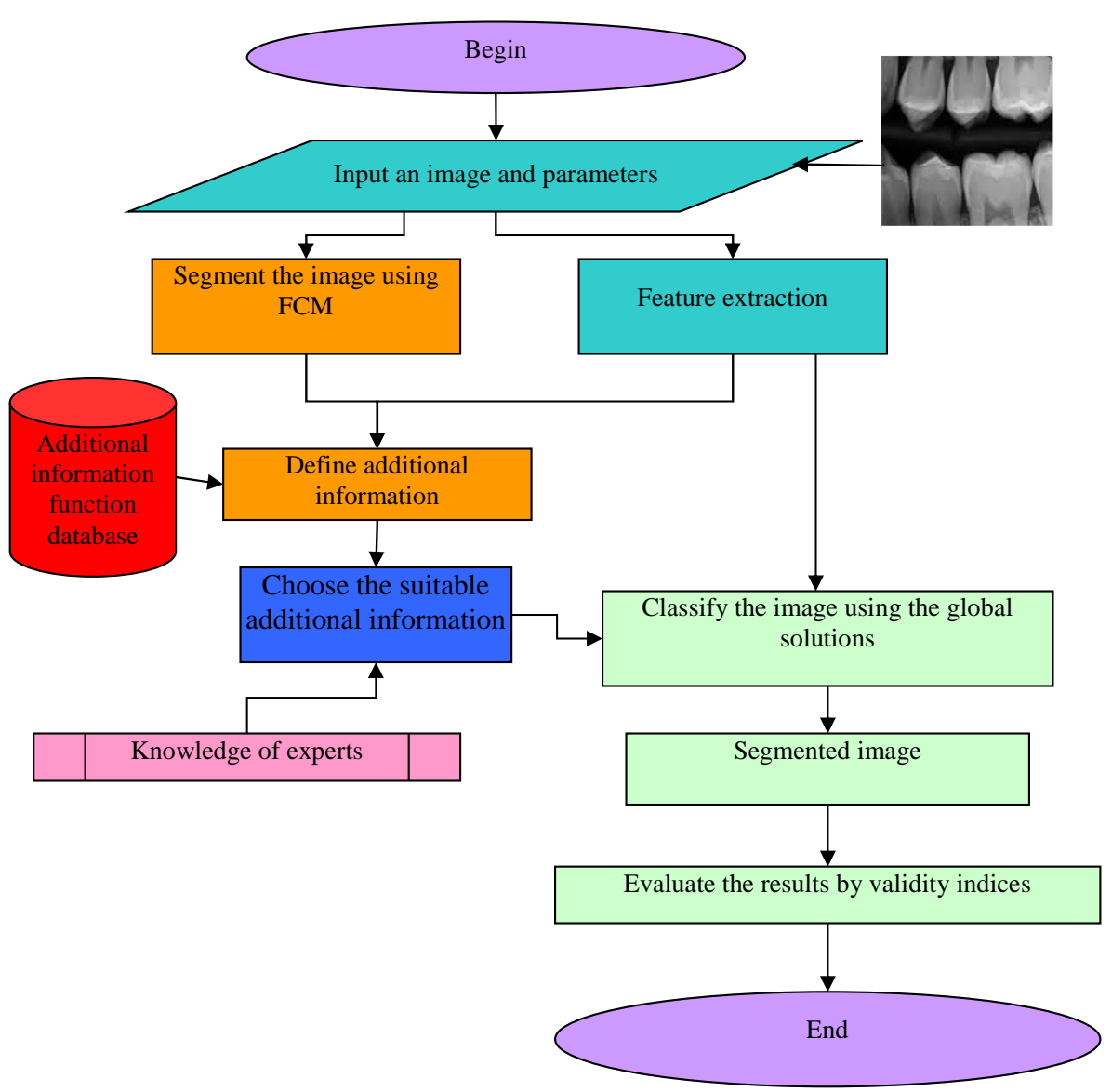

Figure 1: The SSFC-FSAI method

b) Bell function:

$$
B_{k j}=\frac{1}{1+\left|\frac{\left\|x_{k}-v_{j}\right\|}{a}\right|^{2 b}}, \quad j=\overline{1, C}, k=\overline{1, N}
$$

where $\mathrm{a}, \mathrm{b}$ are parameters, $\mathrm{x}_{j}$ : is the data of $\mathrm{j}^{\text {th }}$ pixel, $v_{i}$ : is the center of $\mathrm{i}^{\text {th }}$ cluster (defined from FCM).

$$
\overline{u_{k j}}=\alpha \frac{B_{k j}}{\sum_{i=1}^{C} B_{k i}+\min _{i=\overline{1, C}}\left\{B_{k i}\right\}}, \alpha \in[0,1], j=\overline{1, C}, k=\overline{1, N}
$$

c) Sigmoid function:

$$
S_{k j}=\frac{1}{1+\exp \left(-a\left(\left\|x_{k}-v_{j}\right\|\right)\right)}, j=\overline{1, C}, k=\overline{1, N}
$$


where $a$ is the parameter, $x_{j}$ : is the data of $j^{\text {th }}$ pixel, $v_{i}$ : is the center of $\mathrm{i}^{\text {th }}$ cluster (defined from $\mathrm{FCM})$

$$
\bar{u}_{k j}=\alpha \frac{S_{k j}}{\sum_{i=1}^{C} S_{k i}+\min _{i=\overline{1, C}}\left\{S_{k i}\right\}}, \alpha \in[0,1], j=\overline{1, C}, k=\overline{1, N}
$$

d) Hyperbolic sine function:

$$
\operatorname{Sin}_{k j}=\frac{e^{2\left\|x_{k}-v_{j}\right\|}-1}{2 e^{\left\|x_{k}-v_{j}\right\|}}, j=\overline{1, C}, k=\overline{1, N}
$$

where $x_{j}$ : is the data of $j^{\text {th }}$ pixel, $v_{i}$ : is the center of $\mathrm{i}^{\text {th }}$ cluster (defined from FCM)

$$
\bar{u}_{k j}=\alpha \frac{\operatorname{Sin}_{j k}}{\sum_{i=1}^{C} \operatorname{Sin}_{i k}+\min _{i=\overline{1, C}}\left\{\operatorname{Sin}_{i k}\right\}}, \alpha \in[0,1], j=\overline{1, C}, k=\overline{1, N}
$$

e) Gudermannian function:

$$
G D_{k j}=2 * \arctan \left(e^{\left\|x_{k}-v_{j}\right\|}\right)-\frac{1}{2} \pi, j=\overline{1, C}, k=\overline{1, N}
$$

where $x_{j}$ : is the data of $j^{\text {th }}$ pixel, $v_{i}$ : is the center of $\mathrm{i}^{\text {th }}$ cluster (defined from FCM)

$$
\bar{u}_{k j}=\alpha \frac{G D_{k j}}{\sum_{i=1}^{C} G D_{k i}+\min _{i=\overline{1, C}}\left\{G D_{k i}\right\}}, \alpha \in[0,1], j=\overline{1, C}, k=\overline{1, N}
$$

f) Fresnel function:

$$
F_{k j}=\int_{0}^{\left\|x_{k}-v_{j}\right\|} \cos \left(t^{2}\right) d t, j=\overline{1, C}, k=\overline{1, N}
$$

where $x_{j}$ : is the data of $\mathrm{j}^{\text {th }}$ pixel, $v_{i}$ : is the center of $\mathrm{i}^{\text {th }}$ cluster (defined from $\mathrm{FCM}$ )

$$
\bar{u}_{k j}=\alpha \frac{F_{k j}}{\sum_{i=1}^{C} F_{k i}+\min _{i=1, C}\left\{F_{k i}\right\}}, \alpha \in[0,1], j=\overline{1, C}, k=\overline{1, N}
$$

g) The triangle wave function:

$$
T_{k j}=\frac{2}{a}\left(\left\|x_{k}-v_{j}\right\|-a\left\lfloor\frac{\left\|x_{k}-v_{j}\right\|}{a}+\frac{1}{2}\right\rfloor\right)(-1)^{\left\lfloor\frac{\left\|x_{k}-v_{j}\right\|}{a}+\frac{1}{2}\right\rfloor}, j=\overline{1, C}, k=\overline{1, N}
$$

where a is the parameter, $\mathrm{x}_{j}$ : is the data of $\mathrm{j}^{\text {th }}$ pixel, $\mathrm{v}_{i}$ : is the center of $\mathrm{i}^{\text {th }}$ cluster (defined from FCM)

$$
\bar{u}_{k j}=\alpha \frac{T_{k j}}{\sum_{i=1}^{C} T_{k i}+\min _{i=\overline{1, C}}\left\{T_{k i}\right\}}, \alpha \in[0,1], j=\overline{1, C}, k=\overline{1, N}
$$


h) The mixed function: exactly the function of SSFC-FS in $(4,5)$.

$$
\begin{aligned}
\bar{u}_{k j} & =\left\{\begin{array}{lll}
\alpha u_{1}, & \text { when } & u_{1} \geq u_{2} \\
\alpha u_{2}, & \text { when } & u_{1}<u_{2}
\end{array} \quad, \alpha \in[0,1], j=\overline{1, C}, k=\overline{1, N}\right. \\
u_{2} & =\frac{\sum_{i=1}^{l} w_{i}}{\max \left\{\sum_{i=1}^{l} w_{i}\right\}}
\end{aligned}
$$

i) The spatial function:

$$
\bar{u}_{j k}=\left\{\begin{array}{ll}
\alpha u_{2} & k h i \quad u_{i k}=\max _{i=1, C}\left(u_{i k}\right) \\
0 & k h i \quad u_{i k} \neq \max _{i=\overline{1, C}}\left(u_{i k}\right)
\end{array} \quad, \alpha \in[0,1], j=\overline{1, C}, k=\overline{1, N}\right.
$$

where $u_{2}$ is defined from feature values in each pixel of the images.

j) The fuzzy clustering function:

$$
\bar{u}_{k j}=\left\{\begin{array}{lll}
\alpha u_{k j} & k h i & u_{k i}=\max _{i=1, C}\left(u_{k i}\right) \\
0 & k h i & u_{k i} \neq \max _{i=\overline{1, C}}\left(u_{k i}\right)
\end{array} \quad, \alpha \in[0,1], j=\overline{1, C}, k=\overline{1, N}\right.
$$

$u_{k j}$ is obtained by using FCM.

\subsection{Choosing the suitable function}

To determine the best fit membership matrix for a given dental image, the following steps are defined: Step 1: Use FCM to segment an input image to get U, V matrices.

Step 2: Calculate the IFV function - a typical validity index for clustering [18] defined below.

$$
\begin{aligned}
I F V & =\frac{1}{C} \sum_{j=1}^{C}\left\{\frac{1}{N} \sum_{k=1}^{N} \bar{u}_{k j}^{2}\left[\log _{2} C-\frac{1}{N} \sum_{k=1}^{N} \log _{2} \bar{u}_{k j}\right]^{2}\right\} \times \frac{S D_{\max }}{\overline{\sigma_{D}}} \\
S D_{\max } & =\max _{k \neq j}\left\|V_{k}-V_{j}\right\|^{2} \\
\overline{\sigma_{D}} & =\frac{1}{C} \sum_{j=1}^{C}\left(\frac{1}{N} \sum_{k=1}^{N} d_{k j}\right)
\end{aligned}
$$

Step 3: Compute parameter values of the membership matrix in Section 3.2 having the largest IFV values. For example, consider the Gaussian function in equations $(20,21)$. Find the first order derivation of IFV by $\alpha$ :

$$
\begin{aligned}
\frac{\partial I F V}{\partial \alpha} & =\frac{1}{C} \times \frac{S D_{\max }}{\bar{\sigma}_{D}} \times \frac{1}{N} \sum_{j=1}^{C}\left\{\sum_{k=1}^{N} 2 \bar{u}_{k j} \times \frac{\partial \bar{u}_{k j}}{\partial \alpha} \times\left[\log _{2} C-\frac{1}{N} \sum_{k=1}^{N} \log _{2} \bar{u}_{k j}\right]^{2}\right. \\
& \left.+\sum_{k=1}^{N} \bar{u}_{k j}^{2} \times 2\left[\log _{2} C-\frac{1}{N} \sum_{k=1}^{N} \log _{2} \bar{u}_{k j}\right] \times\left(-\frac{1}{N} \sum_{k=1}^{N} \frac{1}{\bar{u}_{k j} \ln 2} \times \frac{\partial \bar{u}_{k j}}{\partial \alpha}\right)\right\}
\end{aligned}
$$


Using (21) yields

$$
A_{k j}=\frac{\partial \bar{u}_{k j}}{\partial \alpha}=\frac{G_{k j}}{\sum_{i=1}^{C} G_{k i}+\min _{i=1, C}\left\{G_{k i}\right\}}
$$

Let this derivation be equal to zero, it results in two values of $\alpha$ :

$$
\left\{\begin{array} { l } 
{ \operatorname { l o g } _ { 2 } \alpha = B _ { j } } \\
{ \operatorname { l o g } _ { 2 } \alpha = B _ { j } - \frac { 1 } { \operatorname { l n } 2 } }
\end{array} \Leftrightarrow \left\{\begin{array}{l}
\alpha_{1}=2^{B_{j}} \\
\alpha_{2}=2^{B_{j}-\frac{1}{\ln 2}}
\end{array}, \quad B_{j}=\log _{2} C-\frac{1}{N} \sum_{k=1}^{N} A_{k j}, j=1, \ldots C\right.\right.
$$

Find the second order derivation of IFV by $\alpha$ :

$$
\begin{aligned}
\frac{\partial^{2} I F V}{\partial \alpha^{2}}=\frac{1}{C} \times \frac{S D_{\max }}{\overline{\sigma_{D}}} \times \frac{1}{N} & \left\{2 \times \sum_{j=1}^{C}\left(\sum_{k=1}^{N} \bar{u}_{k j}^{2}\right)\left(B_{j}-\log _{2} \alpha\right)\left(B_{j}-\log _{2} \alpha-\frac{1}{\ln 2}\right)\right. \\
& +2 \alpha \times \sum_{j=1}^{C}\left(\sum_{k=1}^{N} \bar{u}_{k j}^{2}\right)\left(-\frac{1}{\alpha \ln 2}\right) \times\left(B_{j}-\log _{2} \alpha-\frac{1}{\ln 2}\right) \\
& \left.+\left(B_{j}-\log _{2} \alpha\right) \times\left(-\frac{1}{\alpha \ln 2}\right)\right\}
\end{aligned}
$$

It follows that

$$
\frac{\partial^{2} I F V}{\partial \alpha^{2}}\left(\alpha_{1}\right)>0, \quad \frac{\partial^{2} I F V}{\partial \alpha^{2}}\left(\alpha_{2}\right)<0
$$

\begin{tabular}{|c|c|}
\hline Input & $\begin{array}{l}\text { Dental X-ray image X; the number of clusters } \mathrm{C} \text {; weight from the expert } \alpha \text {; } \\
\text { threshold } \varepsilon \text {; maxStep }>0\end{array}$ \\
\hline Output & A segmented image determined by membership matrix $\mathrm{U}$ and cluster centers $\mathrm{V}$ \\
\hline \multicolumn{2}{|c|}{ SSFC-FSAI } \\
\hline 1: & nt an input image to get $\mathrm{U}, \mathrm{V} \mathrm{m}$ \\
\hline 2: & Calcul \\
\hline 3: & IFV function by equations $(38-40)$ \\
\hline 4: & $\begin{array}{l}\text { Compute values of the membership matrix in Section } 3.2 \text { having the largest IFV } \\
\text { values. For example, consider the Gaussian function in equations (20-21). Find } \\
\text { the first order derivation of IFV by } \alpha \text { with (46) }\end{array}$ \\
\hline 5: & $\begin{array}{l}\text { Choose this membership matrix and its parameter values as the additional func- } \\
\text { tion }\end{array}$ \\
\hline 6: & Use SSFC-FS for the dental X-ray image with additional function in Step 5 \\
\hline
\end{tabular}

It means that $\alpha_{2}$ is the value that makes IFV get maximum whereas $\alpha_{1}$ makes IFV get minimum.

$$
\alpha_{2}=2^{B_{j}-\frac{1}{\ln 2}}
$$

Step 4: Choose this membership matrix and its parameter values as the additional function.

The SSFC-FSAI method is presented Table 2.

Table 2: SSFC-FSAI algorithm

Computational complexity: The computational complexity depends on the FCM method. In [19], FCM has computational complexity $\mathrm{O}\left(\mathrm{N}^{*}{ }^{*} \mathrm{C} 2 *_{\mathrm{i}}\right)$ where $\mathrm{i}$ is the number of iterations. Thus, 
the computational complexity of our method is approximate to $\mathrm{O}\left(\mathrm{N}^{*} \mathrm{r}^{*} \mathrm{C}{ }^{*} \mathrm{I}\right)$. In this algorithm, the features of dental image are represented through objective functions $\mathrm{J}_{2}$ and $\mathrm{J}_{3}$. The additional functions in equations (35-37) are used for those objective functions. Thus, the technique is specialized for dental images. For other kinds of images, perhaps this technique is not as good as the corresponding methods (The authors have not compared it to others in this case yet).

Example 1. Consider an input $3 \times 3$ image as below.

\begin{tabular}{|c|c|c|}
\hline 59 & 125 & 97 \\
\hline 72 & 142 & 114 \\
\hline 71 & 146 & 116 \\
\hline 49 & 132 & 102 \\
\hline 41 & 130 & 98 \\
\hline 48 & 145 & 112 \\
\hline 47 & 148 & 114 \\
\hline 33 & 139 & 103 \\
\hline 34 & 142 & 106 \\
\hline
\end{tabular}

The weight matrix extracted from this image is:

\begin{tabular}{|l|l|l|l|l|l|l|l|l|}
\hline 0.31 & 0.45 & 0.31 & 0.41 & 0.31 & 0.42 & 0.34 & 0.36 & 0.42 \\
\hline
\end{tabular}

Applying FCM to this image, we obtain the cluster centers and membership matrix:

\begin{tabular}{|l|l|l|}
\hline 70.81 & 143.92 & 114.81 \\
\hline 38.73 & 142.40 & 107.41 \\
\hline 49.49 & 130.18 & 100.03 \\
\hline
\end{tabular}

Cluster centers

\begin{tabular}{|l|l|l|l|l|l|l|l|l|}
\hline 0.12 & 0.99 & 0.98 & 0.01 & 0.04 & 0.14 & 0.16 & 0.03 & 0.02 \\
\hline 0.12 & 0.00 & 0.01 & 0.03 & 0.23 & 0.66 & 0.66 & 0.82 & 0.93 \\
\hline 0.76 & 0.01 & 0.01 & 0.96 & 0.73 & 0.20 & 0.18 & 0.15 & 0.05 \\
\hline
\end{tabular}

Membership matrix

Based on IFV criterion, we select the value of $\alpha$ that makes IFV maximal. Table 3 shows that the $4^{\text {th }}$ function namely Hyperbolic sine is the most suitable for the dental image.

\subsection{Advantages of the new algorithm}

Firstly, SSFC-FSAI is better than SSFC-FS in term of clustering quality since each dental image is processed with a different additional function that is best fit with the image, and hence increasing the overall accuracy.

Secondly, SSFC-FSAI automatically determines the parameter values for the highest quality of the clustering algorithm.

Thirdly, the new parts cooperate with the old ones in a unified framework that supports effectively medical diagnosis. 


\begin{tabular}{|c|c|c|c|c|c|c|c|c|c|c|}
\hline $\mathrm{i}^{\text {th }}$ function & IFV & & & & & $\bar{U}_{i}$ & & & & \\
\hline 1 & 1.7456 & 0.25 & 0.35 & 0.35 & 0.23 & 0.21 & 0.25 & 0.25 & 0.20 & 0.21 \\
\hline & & 0.22 & 0.21 & 0.22 & 0.26 & 0.28 & 0.28 & 0.28 & 0.32 & 0.32 \\
\hline & & 0.30 & 0.23 & 0.21 & 0.29 & 0.30 & 0.24 & 0.23 & 0.28 & 0.26 \\
\hline 2 & 10.212 & 0.11 & 0.98 & 0.98 & 0.01 & 0.04 & 0.12 & 0.14 & 0.03 & 0.02 \\
\hline & & 0.11 & 0.01 & 0.01 & 0.03 & 0.22 & 0.57 & 0.56 & 0.79 & 0.91 \\
\hline & & 0.68 & 0.01 & 0.01 & 0.95 & 0.70 & 0.18 & 0.16 & 0.14 & 0.06 \\
\hline 3 & 1.6093 & 0.25 & 0.24 & 0.24 & 0.26 & 0.25 & 0.25 & 0.25 & 0.25 & 0.25 \\
\hline & & 0.25 & 0.26 & 0.26 & 0.26 & 0.25 & 0.25 & 0.25 & 0.25 & 0.25 \\
\hline & & 0.25 & 0.26 & 0.26 & 0.24 & 0.25 & 0.25 & 0.25 & 0.25 & 0.25 \\
\hline 4 & 133.49 & 0.47 & 0.00 & 0.00 & 1.00 & 1.00 & 0.98 & 0.80 & 1.00 & 1.00 \\
\hline & & 0.53 & 0.99 & 0.92 & 0.00 & 0.00 & 0.00 & 0.00 & 0.00 & 0.00 \\
\hline & & 0.00 & 0.01 & 0.08 & 0.00 & 0.00 & 0.02 & 0.20 & 0.00 & 0.00 \\
\hline 5 & 1.6137 & 0.25 & 0.23 & 0.23 & 0.26 & 0.25 & 0.25 & 0.25 & 0.25 & 0.25 \\
\hline & & 0.25 & 0.27 & 0.27 & 0.26 & 0.25 & 0.25 & 0.25 & 0.25 & 0.25 \\
\hline & & 0.25 & 0.27 & 0.27 & 0.24 & 0.25 & 0.25 & 0.25 & 0.25 & 0.25 \\
\hline 6 & 1.8087 & 0.25 & 0.22 & 0.23 & 0.24 & 0.25 & 0.25 & 0.26 & 0.06 & 0.26 \\
\hline & & 0.25 & 0.28 & 0.27 & 0.23 & 0.25 & 0.25 & 0.24 & 0.45 & 0.24 \\
\hline & & 0.26 & 0.27 & 0.28 & 0.30 & 0.26 & 0.25 & 0.25 & 0.42 & 0.27 \\
\hline 7 & 2.4646 & 0.30 & 0.44 & 0.30 & 0.04 & 0.06 & 0.02 & 0.27 & 0.36 & 0.11 \\
\hline & & 0.22 & 0.09 & 0.30 & 0.41 & 0.31 & 0.42 & 0.05 & 0.05 & 0.06 \\
\hline & & 0.16 & 0.01 & 0.08 & 0.27 & 0.31 & 0.14 & 0.34 & 0.29 & 0.41 \\
\hline 8 & 1.3421 & 0.00 & 0.99 & 0.99 & 0.00 & 0.00 & 0.00 & 0.00 & 0.00 & 0.00 \\
\hline & & 0.00 & 0.00 & 0.00 & 0.00 & 0.00 & 0.66 & 0.66 & 0.82 & 0.93 \\
\hline & & 0.76 & 0.00 & 0.00 & 0.96 & 0.73 & 0.00 & 0.00 & 0.00 & 0.00 \\
\hline 9 & 0.4113 & 0.00 & 0.45 & 0.31 & 0.00 & 0.00 & 0.00 & 0.00 & 0.00 & 0.00 \\
\hline & & 0.00 & 0.00 & 0.00 & 0.00 & 0.00 & 0.42 & 0.34 & 0.36 & 0.42 \\
\hline & & 0.31 & 0.00 & 0.00 & 0.41 & 0.31 & 0.00 & 0.00 & 0.00 & 0.00 \\
\hline 10 & 1.3421 & 0.00 & 0.99 & 0.99 & 0.00 & 0.00 & 0.00 & 0.00 & 0.00 & 0.00 \\
\hline & & 0.00 & 0.00 & 0.00 & 0.00 & 0.00 & 0.66 & 0.66 & 0.82 & 0.93 \\
\hline & & 0.76 & 0.00 & 0.00 & 0.96 & 0.73 & 0.00 & 0.00 & 0.00 & 0.00 \\
\hline
\end{tabular}

Table 3: Values of IFV to choose the best additional information 


\section{EXPERIMENTAL RESULTS}

\subsection{Implemented environment}

The authors describe the experimental environments including:

Implemented tools: the SSFC-FSAI method has been implemented in addition to FCM [1], eSFCM [16], Otsu [12], and SSFC-FS [18] in Matlab 2014 and performed on a VAIO laptop with Core i5 processor. The results are taken and computed as the average of 20 iterations.

Experimental datasets: are taken from Hanoi Medical University, Vietnam including 56 dental images in the period 2014 - 2015. The datasets were uploaded to Matlab Central for sharing.

Cluster validity measurements [14]: For the purpose to measure the performance of algorithms exactly, 8 typical validity indices are used such as: Davies-Bouldin (DB), Simplified Silhouete Width Criterion (SSWC), PBM, IFV, Ball and Hall index (BH), Calinski-Harabasz index (VCR), BanfeldRaftery index (BR), and Difference-like index (TRA). Among all, the lower values of DB and BR are better. Regarding the remains, greater value shows more efficient algorithm.

\subsection{The comparison of accuracy}

Table 4 shows means and variances of each cluster validity criterion for all algorithms. It is clear that SSFC-FSAI gets the best performance in three criteria PBM, BH, BR whilst SSFC-FS has the best value in other three criteria including SSWC, VRC and TRA. In DB and IFV criteria, SSFC-FSAI obtains better value than SSFC-FS. This shows that SSFC-FSAI is able to adapt with datasets more effectively and dynamically than SSFC-FS and other algorithms.

\begin{tabular}{cccccc}
\hline Method & FCM & OTSU & eSFCM & SSFC-FS & SSFC-FSAI \\
\hline \hline PBM & 34590.65 & 39438.83 & 30357.89 & 49523.87 & $\mathbf{5 4 5 0 9 . 2 3}$ \\
& $\pm 5.54 \mathrm{E}+08$ & 857679906 & $\pm 5.69 \mathrm{E}+08$ & $\pm 2.34 \mathrm{E}+09$ & $\pm 0.98 \mathrm{E}+09$ \\
\hline DB & $\mathbf{0 . 6 5 8}$ & 0.846 & 0.708 & 0.832 & 0.823 \\
\pm 0.006 & \pm 1.034 & \pm 0.01 & \pm 0.045 & \pm 0.044 & \\
\hline IFV & 30.34 & Inf & $\mathbf{4 4 9 . 2 5}$ & 50.87 & 89.89 \\
& \pm 245.41 & & \pm 77655.09 & \pm 562.73 & \pm 372.2 \\
\hline SSWC & 0.629 & 0.656 & 0.646 & $\mathbf{1 . 2 6 3}$ & 0.983 \\
& \pm 0.008 & \pm 0.01 & \pm 0.01 & \pm 4.36 & \pm 3.23 \\
\hline VRC & 8773901 & 6422160 & 8657364 & $\mathbf{1 1 5 3 5 2 4 4}$ & 10662842 \\
& $\pm 1.67 \mathrm{E}+14$ & $\pm 1.68 \mathrm{E}+14$ & $\pm 1.69 \mathrm{E}+14$ & $\pm 0.83 \mathrm{E}+14$ & $\pm 1.32 \mathrm{E}+14$ \\
\hline BH & 1466.96 & 838.30 & 1520.20 & 2109.98 & $\mathbf{2 3 2 7 . 3 7}$ \\
& \pm 40315.4 & \pm 90125.07 & \pm 50465.31 & \pm 178232.9 & \pm 186227.7 \\
\hline BR & $-1.9 \mathrm{E}+07$ & $-1.5 \mathrm{E}+07$ & $-1.9 \mathrm{E}+07$ & $-2.3 \mathrm{E}+07$ & $\mathbf{- 2 . 4 E}+\mathbf{0 7}$ \\
& $\pm 1.64 \mathrm{E}+14$ & $\pm 6.85 \mathrm{E}+14$ & $\pm 1.51 \mathrm{E}+14$ & $\pm 0.98 \mathrm{E}+14$ & $\pm 1.18 \mathrm{E}+14$ \\
\hline TRA & $5.09 \mathrm{E}+09$ & $2.43 \mathrm{E}+09$ & $5.34 \mathrm{E}+09$ & $\mathbf{6 . 7 8 E}+\mathbf{0 9}$ & $5.87 \mathrm{E}+09$ \\
& $\pm 8.51 \mathrm{E}+18$ & $\pm 4.18 \mathrm{E}+18$ & $\pm 7.66 \mathrm{E}+18$ & $\pm 6.08 \mathrm{E}+18$ & $\pm 8.00 \mathrm{E}+18$ \\
\hline
\end{tabular}

Table 4: Means and variances of the criteria for all algorithms on the real dataset (Bold values indicate the better in a row)

From this table, the best result in a row is set as value 1. The authors calculate the number of times that the best algorithm is better than others in the same row and present the results in Table 5 . 


\begin{tabular}{lccccc}
\hline Hits more & FCM & OTSU & eSFCM & SSFC-FS & SSFC-FSAI \\
\hline PBM & 1.58 & 1.38 & 1.80 & 1.10 & 1.00 \\
\hline DB & 1.00 & 1.29 & 1.08 & 1.26 & 1.25 \\
\hline IFV & 14.81 & Inf & 1.00 & 8.83 & 5.00 \\
\hline SSWC & 2.01 & 1.93 & 1.96 & 1.00 & 1.28 \\
\hline VRC & 1.31 & 1.80 & 1.33 & 1.00 & 1.08 \\
\hline BH & 1.59 & 2.78 & 1.53 & 1.10 & 1.00 \\
\hline BR & 1.26 & 1.60 & 1.26 & 1.04 & 1.00 \\
\hline TRA & 1.33 & 2.79 & 1.27 & 1.00 & 1.16 \\
\hline
\end{tabular}

Table 5: Performance comparison of all algorithms on the real dataset (value 1 indicates the best in a row)

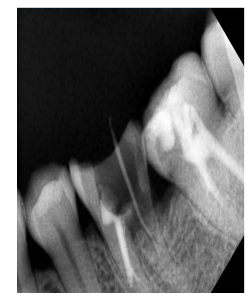

(a)

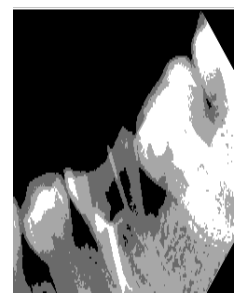

(b)

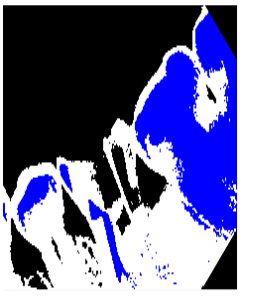

(c)

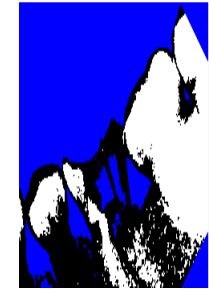

(d)

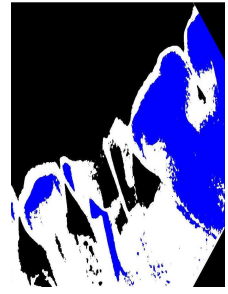

(e)

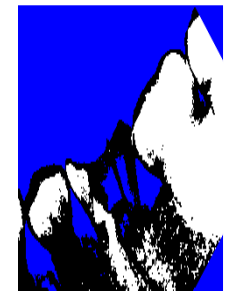

(f)

Figure 2: (a) A dental image; (b) Otsu; (c) FCM; (d) eSFCM; (e) SSFC-FS; (f) SSFC-FSAI

Figure 2 gives the visual result of dental X-ray image segmentation of a dental image including OTSU, FCM, eSFCM, SSFC-FS, and SSFC-FSAI.

In order to know how different membership matrix in additional information would affect the clustering quality of SSFC-FSAI, the method is validated on an image and by different (manual) membership matrices. The average results are stated in Table 6 .

\begin{tabular}{cccccc}
\hline & $\bar{U}_{1}$ & $\bar{U}_{2}$ & $\bar{U}_{3}$ & $\bar{U}_{4}$ & $\bar{U}_{5}$ \\
\hline PBM & 51378.78 & 36862.877 & 37887.73 & 47643.78 & 46383.84 \\
\hline DB & 0.783 & $\mathbf{0 . 7 3 8}$ & 0.836 & 0.823 & 0.801 \\
\hline IFV & 104.98 & 115.82 & 100.28 & 99.82 & 86.83 \\
\hline SSWC & 0.893 & $\mathbf{0 . 9 6 3}$ & 0.824 & 0.927 & 0.903 \\
\hline VRC & 9972637 & 9236544 & $\mathbf{1 1 4 7 2 3 4 3}$ & 10375234 & 7872473 \\
\hline BH & 1576.83 & 1198.32 & $\mathbf{2 3 9 8 . 3 8}$ & 2215.82 & 1973.87 \\
\hline BR & -12276432 & -17762553 & -16827832 & -16376284 & -11473473 \\
\hline TRA & 9382468643 & 8276724283 & $\mathbf{9 3 8 4 8 6 4 3 3 4}$ & 9082534578 & 7355264545 \\
\hline
\end{tabular}




\begin{tabular}{cccccc}
\hline & $\bar{U}_{6}$ & $\bar{U}_{7}$ & $\bar{U}_{8}$ & $\bar{U}_{9}$ & $\bar{U}_{10}$ \\
\hline PBM & 42384.87 & 52784.27 & 40978.78 & 47386.82 & $\mathbf{5 6 7 8 4 . 8 9}$ \\
\hline DB & 0.767 & 0.869 & 0.750 & 0.879 & 0.934 \\
\hline IFV & $\mathbf{1 6 5 . 7 8}$ & 156.32 & 97.23 & 87.98 & 88.99 \\
\hline SSWC & 0.890 & 0.902 & 0.742 & 0.779 & 0.813 \\
\hline VRC & 10208732 & 9722574 & 9638374 & 9453635 & 9243746 \\
\hline BH & 1753.78 & 2083.46 & 1989.74 & 1929.58 & 1863.38 \\
\hline BR & -15376746 & -17623643 & -18736354 & $\mathbf{- 1 9 8 7 3 4 3 8}$ & -19837455 \\
\hline TRA & 8937427838 & 8462732432 & 8352644536 & 8754653643 & 8848637346 \\
\hline
\end{tabular}

Table 6: Using different additional information functions for SSFC-FSAI
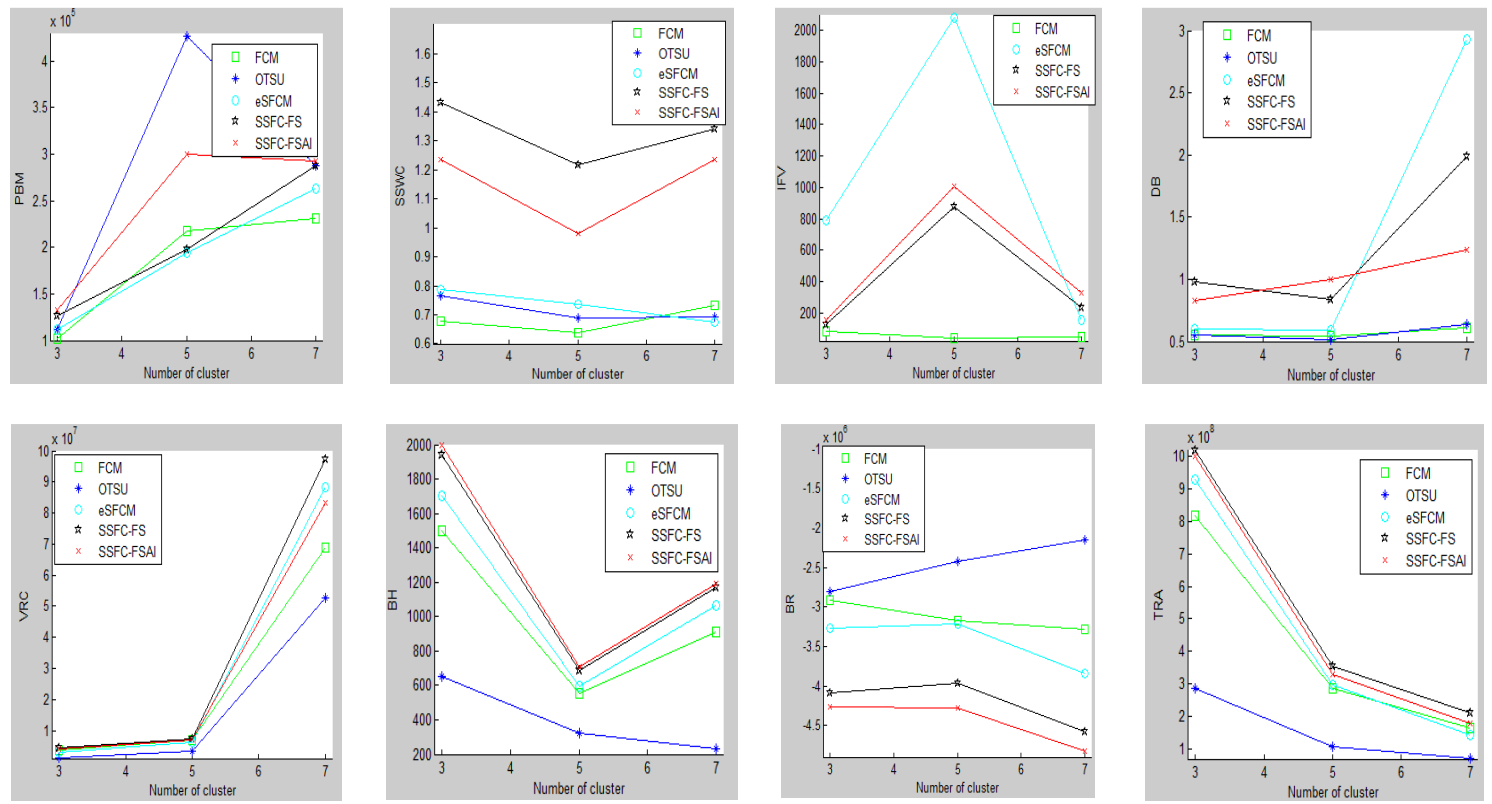

Figure 3: The accuracies of methods by the number of clusters

\section{CONCLUSIONS}

In this paper, the authors concentrate on the dental X-ray image segmentation problem and present a new dynamic semi-supervised fuzzy clustering algorithm called SSFC-FSAI. The new contributions are summarized as follows. Firstly, 10 membership functions are proposed in Section 3.2 to be the functional warehouse for additional information of SSFC-FSAI. Secondly, a new procedure in SSFCFSAI to choose a suitable membership function and its parameter values for a given dental image is presented in Section 3.3. Thirdly, the experimental validation on 56 dental X-ray images of Hanoi University of Medical, Vietnam in $2014-2015$ is taken to validate the efficiency of the new method. The achieved results show the characteristics of the algorithm and suggested appropriate experimental environmental settings for further validation. 
Further works of this research will investigate the second part of medical diagnosis, that is to say, the disease matching problem from segmented image.

\section{APPENDIX}

Matlab source codes of all algorithms and experimental data can be found at the URL: https://www.mathworks.com/matlabcentral/fileexchange/53313-the-best-additional-informationfunction-for-semi-supervised-fuzzy-clustering.

\section{REFERENCES}

[1] J. C. Bezdek, R. Ehrlich, and W. Full, "Fcm: The fuzzy c-means clustering algorithm," Computers $\& 3$ Geosciences, vol. 10, no. 2, pp. 191-203, 1984.

[2] A. Bouchachia and W. Pedrycz, "Data clustering with partial supervision," Data Mining and Knowledge Discovery, vol. 12, no. 1, pp. 47-78, 2006.

[3] A. Chen, T. Wittman, A. Tartakovsky, and A. Bertozzi, "Image segmentation through efficient boundary sampling," in SAMPTA '09, 2009, pp. Special-session.

[4] S. Gould, T. Gao, and D. Koller, "Region-based segmentation and object detection," in Advances in neural information processing systems, 2009, pp. 655-663.

[5] T. Leung and J. Malik, "Contour continuity in region based image segmentation," in Computer VisionECCV'98. Springer, 1998, pp. 544-559.

[6] S. Li, T. Fevens, A. Krzyżak, and S. Li, "An automatic variational level set segmentation framework for computer aided dental x-rays analysis in clinical environments," Computerized Medical Imaging and Graphics, vol. 30, no. 2, pp. 65-74, 2006.

[7] H. Narkhede, "Review of image segmentation techniques," Int. J. Sci. Mod. Eng, vol. 1, no. 5461, p. $28,2013$.

[8] J. Nayak, B. Naik, and H. Behera, "Fuzzy c-means (fcm) clustering algorithm: A decade review from 2000 to 2014," in Computational Intelligence in Data Mining-Volume 2. Springer, 2015, pp. 133-149.

[9] L. T. Ngo, D. S. Mai, and W. Pedrycz, "Semi-supervising interval type-2 fuzzy c-means clustering with spatial information for multi-spectral satellite image classification and change detection," Computers \& Geosciences, vol. 83, pp. 1-16, 2015.

[10] O. Nomir and M. Abdel-Mottaleb, "A system for human identification from x-ray dental radiographs," Pattern Recognition, vol. 38, no. 8, pp. 1295-1305, 2005.

[11] N. Otsu, "A threshold selection method from gray-level histograms," Automatica, vol. 11, no. 285-296, pp. 23-27, 1975.

[12] A. E. Rad, M. S. M. Rahim, and A. Norouzi, "Level set and morphological operation techniques in application of dental image segmentation," International Scholarly and Scientific Research 8 Innovation, vol. 8, no. 4, pp. 177-180, 2014.

[13] E. Yasunori, H. Yukihiro, Y. Makito, and M. Sadaaki, "On semi-supervised fuzzy c-means clustering," in IEEE International Conference on Fuzzy Systems. FUZZ-IEEE 2009. IEEE, 2009, pp. 1119-1124. 
[14] X. Yin, T. Shu, and Q. Huang, "Semi-supervised fuzzy clustering with metric learning and entropy regularization," Knowledge-Based Systems, vol. 35, pp. 304-311, 2012.

[15] G. Zhu, S. Zhang, Q. Zeng, and C. Wang, "Boundary-based image segmentation using binary level set method," Optical Engineering, vol. 46, no. 5, pp. 050 501-050 501, 2007.

[16] T. M. Tuan, T. T. Ngan, and L. H. Son, "A novel semi-supervised fuzzy clustering method based on interactive fuzzy satisficing for dental x-ray image segmentation," Applied Intelligence, in presss.

[17] C. Mohan and H. T. Nguyen, "An interactive satisficing method for solving multiobjective mixed fuzzy-stochastic programming problems," Fuzzy sets and Systems, vol. 117, no. 1, pp. 61-79, 2001.

[18] L. Vendramin, R. J. Campello, and E. R. Hruschka, "Relative clustering validity criteria: A comparative overview," Statistical Analysis and Data Mining: The ASA Data Science Journal, vol. 3, no. 4, pp. 209-235, 2010.

[19] S. Ghosh and S. K. Dubey, "Comparative analysis of k-means and fuzzy c-means algorithms," IJACSA, vol. 4, pp. 35-38, 2013.

Received on October $04-2014$

Revised on August 31 - 2015 